\title{
EchoGéo
}

\section{La réélection de Lula, changement de base}

\author{
Hervé Théry et André Nagy
}

\section{(Q) OpenEdition}

Journals

Édition électronique

URL : https://journals.openedition.org/echogeo/2129

DOI : 10.4000/echogeo.2129

ISSN : 1963-1197

\section{Éditeur}

Pôle de recherche pour l'organisation et la diffusion de l'information géographique (CNRS UMR 8586)

Référence électronique

Hervé Théry et André Nagy, «La réélection de Lula, changement de base », EchoGéo [En ligne], 3 | 2007, mis en ligne le 18 décembre 2007, consulté le 01 août 2021. URL : http://

journals.openedition.org/echogeo/2129; DOI : https://doi.org/10.4000/echogeo.2129

Ce document a été généré automatiquement le 1 août 2021

EchoGéo est mis à disposition selon les termes de la licence Creative Commons Attribution - Pas d'Utilisation Commerciale - Pas de Modification 4.0 International (CC BY-NC-ND) 


\title{
La réélection de Lula, changement de base
}

\author{
Hervé Théry et André Nagy
}

1 Luiz Inácio da Silva, plus connu sous son surnom de Lula (désormais intégré officiellement à son nom), a été réélu président de la République brésilienne, le 29 octobre 2006, après avoir manqué de peu l'élection au premier tour, le 1er octobre. Cette victoire a été célébrée dans bon nombre pays du monde, en France notamment, commme le renouvellement de la confiance du pays en son président, issu de la classe ouvrière et du mouvement syndical, malgré une série de scandales politico-financiers qui ont marqué les trois dernières années de son premier mandat.

2 C'est en partie vrai, une partie de 58 millions de voix qui se sont portées sur son nom sont celles de partisans de longue date, mais on a moins remarqué hors du Brésil que sa base électorale principale avait changé, socialement et géographiquement: appuyé traditionnellement par les régions industrielles du Sud, ce qui était conforme à son parcours personnel antérieur, il a été cette fois porté par le Nord et le Nordeste, les régions pauvres, voire sous-développées, du pays, comme le montre une série de cartes ${ }^{1}$ fondées sur l'analyse des résultats électoraux.

Les candidats en présence au second tour étaient Lula et Geraldo Alckmin, du PSDB (parti social-démocrate) allié au PFL (droite) : ce second tour a confirmé la bipolarisation de la vie politique brésilienne, les votes des « petits » candidats du premier tour se reportant principalement sur Lula ${ }^{2}$.

Tableau 1

\begin{tabular}{|l|l|l|l|l|l|}
\hline Candidat & Parti & Votes & $\%$ & Alliance \\
\hline $1^{\text {er }}$ tour & PT & 46662365 & 48,61 & PT / PRB / PC do B \\
\hline \hline Luiz Inácio Lula da Silva &
\end{tabular}




\begin{tabular}{|l|l|l|l|l|}
\hline Geraldo José Rodrigues Alckmin Filho & PSDB & 39968369 & 41,64 & PSDB / PFL \\
\hline Heloísa Helena Lima de Moraes Carvalho & PSOL & 6575393 & 6,85 & PSTU / PCB / PSOL \\
\hline Cristovam Ricardo Calvacanti Buarque & PDT & 2538844 & 2,65 & \\
\hline Ana Maria Teixeira Rangel & PRP & 126404 & 0,13 & \\
\hline José Maria Eymael & PSDC & 63294 & 0,07 & \\
\hline Luciano Caldas Bivar & PSL & 62064 & 0,07 & \\
\hline $2^{\mathbf{e}}$ Tour & & & & \\
\hline Luiz Inácio Lula da Silva & PT & 58295042 & 60,83 & PT / PRB / PC do B \\
\hline \hline Geraldo José Rodrigues Alckmin Filho & PSDB & 37543178 & 39,17 & PSDB / PFL \\
\hline
\end{tabular}

$4 \quad$ Un des aspects les plus étonnants de cette élection est qu'à la bipolarisation politique a correspondu une bipolarisation territoriale, Lula apparaissant comme l'élu des régions pauvres du Nord amazonien et du Nordeste, alors que son concurrent était appuyé par le Sud et le Sudeste, plus développés. Cela n'allait pas de soi, et constitue même une rupture avec l'histoire personnelle de Lula. Il est certes né dans le Nordeste (le octobre 1945 à Caetés, commune de Garanhuns, dans le Pernambuco), mais il en est parti à l'âge de sept ans, avec ses parents dans leur migration, en pau de arara (littéralement " perchoir à perroquets ", un camion sommairement aménagé) vers São Paulo, comme tant d'autres Nordestins à cette époque. Il a commencé à y travailler très jeune, à douze ans (employé dans une teinturerie, puis cireur de chaussures, puis employé de bureau) et $\mathrm{y}$ a fait l'essentiel de sa carrière syndicale et politique, au syndicat des métallurgistes, puis au Parti des Travailleurs (PT), dont il est l'un des fondateurs. Sa base électorale était bien dans le Sudeste et le Sud, jusqu'à l'élection victorieuse de 2002, après les trois tentatives de 1990, 1994 et 1998. Toutes trois avaient été marquées par cette implantation dans les régions développées, où se situent les villes industrielles et les entreprises modernes, les principales universités et autres bases électorales du PT. Il est donc étonnant de voir qu'en 2006, tant en nombre d'électeurs qu'en proportion de ceux-ci dans l'électorat, Lula réalise ses meilleurs scores dans le Nordeste et en Amazonie, alors que dans le Sud-Sudeste il est devancé par son adversaire. 


\section{Alckmin au deuxième tour}

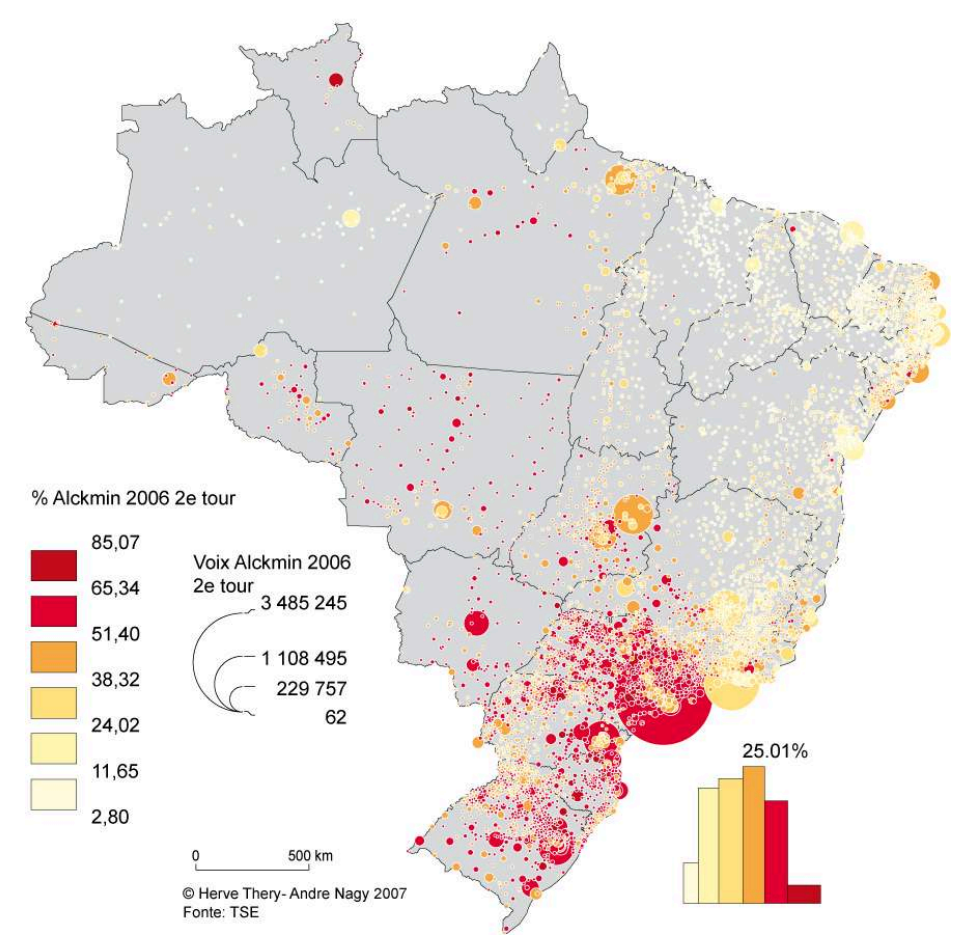

5 Celui-ci, Geraldo José Rodrigues Alckmin Filho (né à Pindamonhangaba, État de São Paulo, le 7 novembre 1952), médecin anesthésiste de formation, a fait toute sa carrière dans l'Etat de São Paulo: conseiller municipal puis maire de sa ville natale, deputé de l'État de São Paulo, député federal, vice-gouverneur de Mario Covas, le très populaire gouverneur de São Paulo (jusqu'à la mort de celui-ci), puis gouverneur pour le reste du mandat, puis élu gouverneur en 2001. Bon administrateur, discret, il est dépourvu de charisme, au point d'avoir été surnommé méchamment " picolé de chuchu» (quelque chose comme «sorbet de navet»). On notera que tout le monde l'appelle par son nom de famille, Alckmin, dans un pays où l'usage du prénom est la norme... La répartition des votes qui se sont portés sur ce nom - ou plutôt sur celui du candidat de l'alliance PSDB-PFL et de principal opposant à Lula - montre sans surprise une concentration dans le Sudeste - notamment la ville de São Paulo, son fief, ainsi que quelques succès dans les régions pionnières d'Amazonie et du Mato Grosso. 


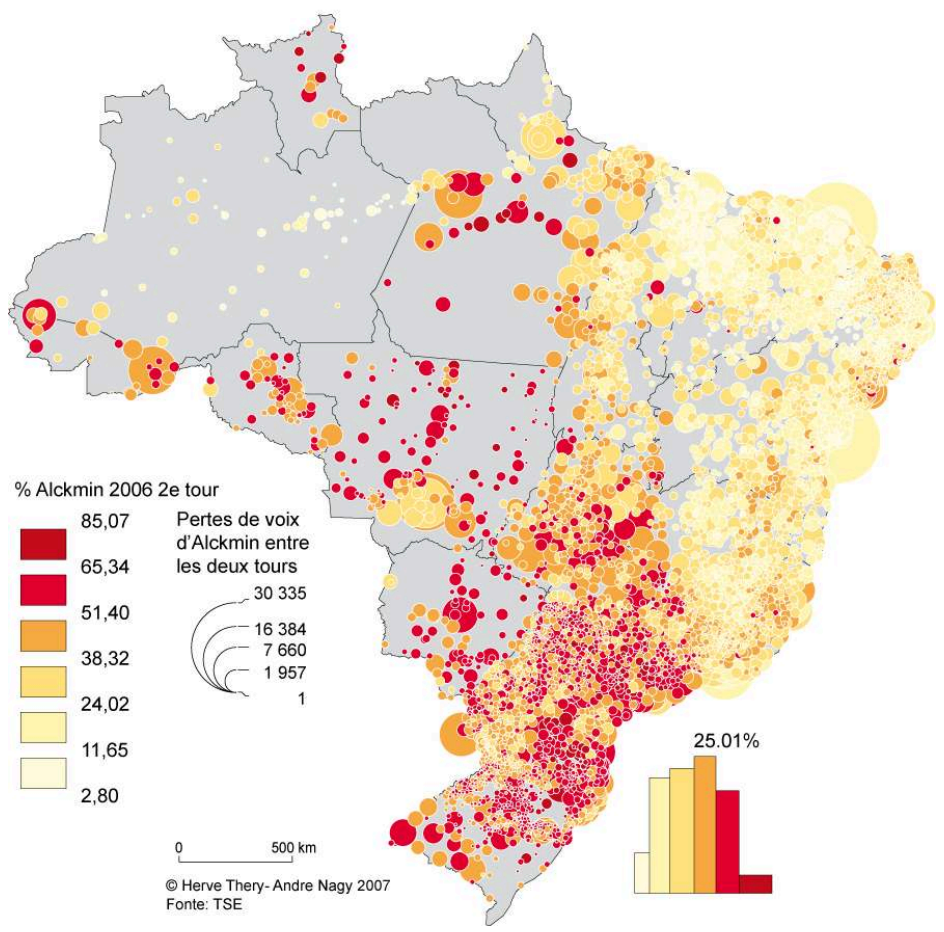

6 Plus étonnant est le fait - rare - qu'il ait perdu entre les deux tours près de deux millions et demi de voix (exactement 2425191 selon le Tribunal Superior Eleitoral), alors que, au Brésil comme ailleurs, les candidats encore présents au deuxième tour rassemblent d'habitude, outre les voix de leurs partisans du premier tour, une partie de celles qui avaient appuyé des candidats éliminés. Malgré les consignes donnés par quelques-uns de ceux-ci, très opposés à Lula, Alckmin n'a donc visiblement pas été un candidat de rassemblement, et il semble avoir surtout perdu des votes protestataires du premier tour: de nombreux électeurs habituels du PT, déçus par l'inefficacité et les dérives du parti une fois arrivés aux affaires, mais qui n'ont pas voulu pour autant porter au pouvoir son adversaire. 
Illustration 3 - Lula au deuxième tour

\section{Lula au deuxième tour}

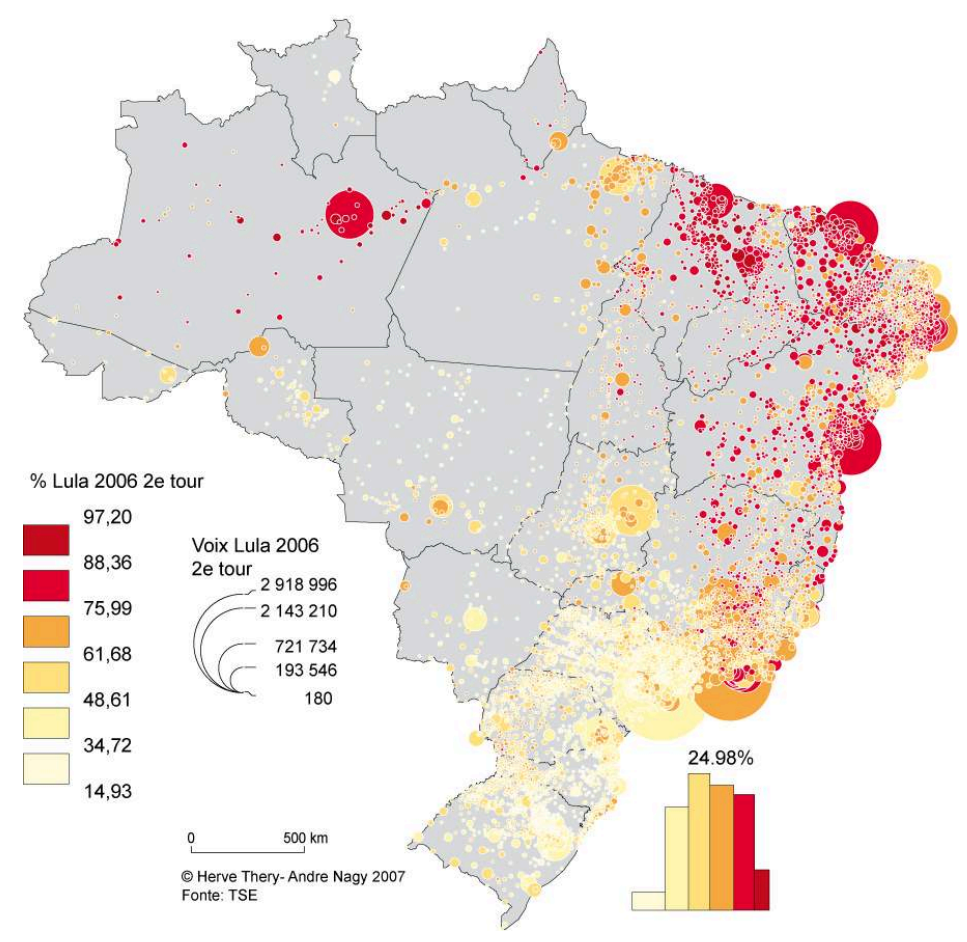

7 Le changement de la base électorale de Lula apparaît nettement si l'on fait la comparaison de son scores avec celui de l'élection de 2002 : en comparant les voix qui se sont portée sur lui en 2002 et en 2006, on fait apparaître qu'il n'a perdu des électeurs que dans le Sud-Sudeste (et le Roraima, à l'extrême nord), et en a gagné principalement dans le Nord et le Nordeste. Les quelques pertes qu'il a subies dans le Nordeste sont dues à la candidature d'Heloisa Helena, une dissidente de gauche du PT, et ses rares gains dans la moitié nord du pays se situent dans le Mato Grosso, dans la région de développement du front pionnier du soja, dont les producteurs, c'est le moins qu'on puisse dire, ne sont pas très à gauche. 


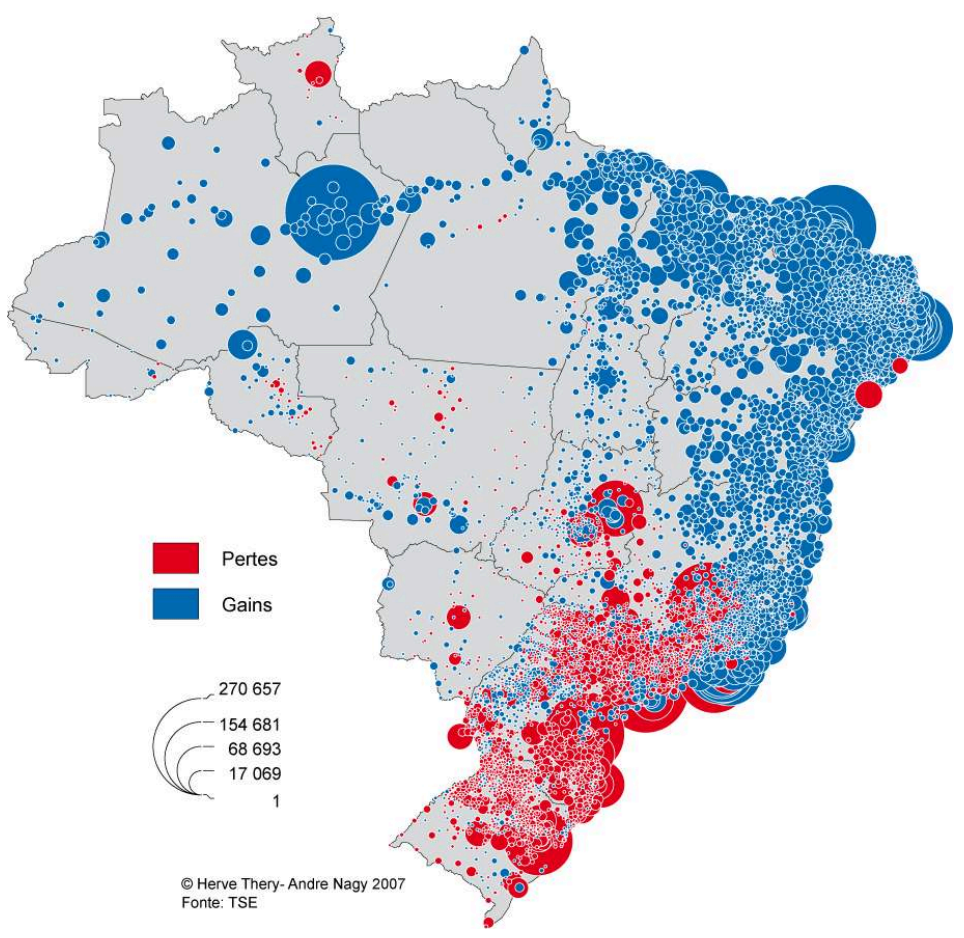

Pourquoi ce vote massif des régions pauvres en faveur de Lula? Une partie de l'explication est à chercher dans le très grand «légitimisme» de ces régions, qui votent depuis longtemps pour le pouvoir en place, dont elles dépendent étroitement pour toute une série d'aides: par exemple, à la fin du régime militaire (1964-1985) le Nordeste était le dernier grand bastion qui lui restait fidèle. Un comportement qui rappelle - mutatis mutandis - celui des Dom-Tom en France.

Une autre explication, sans doute la principale, est la reconnaissance des bénéficiaires de la Bolsa familia, l'aide versée aux familles les plus pauvres. Inventée sous le gouvernement précédent mais amplifiée et systématisée par le gouvernement Lula, cette aide peut paraitre dérisoire (autour de 60 Reais par famille et par mois, soit environ 22 euros), mais elle peut être décisive pour des populations vivant avec moins de 120 Reais par mois (l'équivalent de 45 euros), à qui cette aide est réservée.

Versées à date fixe, payées par versement sur une carte de paiement (semblable à une carte de crédit, ce qui contribue à donner à ses titulaires une nouvelle dignité), ces aides ont concerné en 2006 plus de 11 millions de familles, précisément concentrées dans ces régions déprimées: la région Nordeste reçoit $69,1 \%$ du total, le nombre de bénéficiaires dans chaque commune y varie de $13 \%$ à $45 \%$ du total de la population Viennent ensuite le Sudeste $(19,1 \%)$, le Nord (8\%), le Centro-Oeste $(2,4 \%)$ et le Sud $(1,4 \%)$. On comprend donc que les régions les plus pauvres du pays, Nordeste et haute Amazonie, aient voté pour qui leur assurait que cette modeste manne continuerait à leur arriver pour cinq ans de plus. 


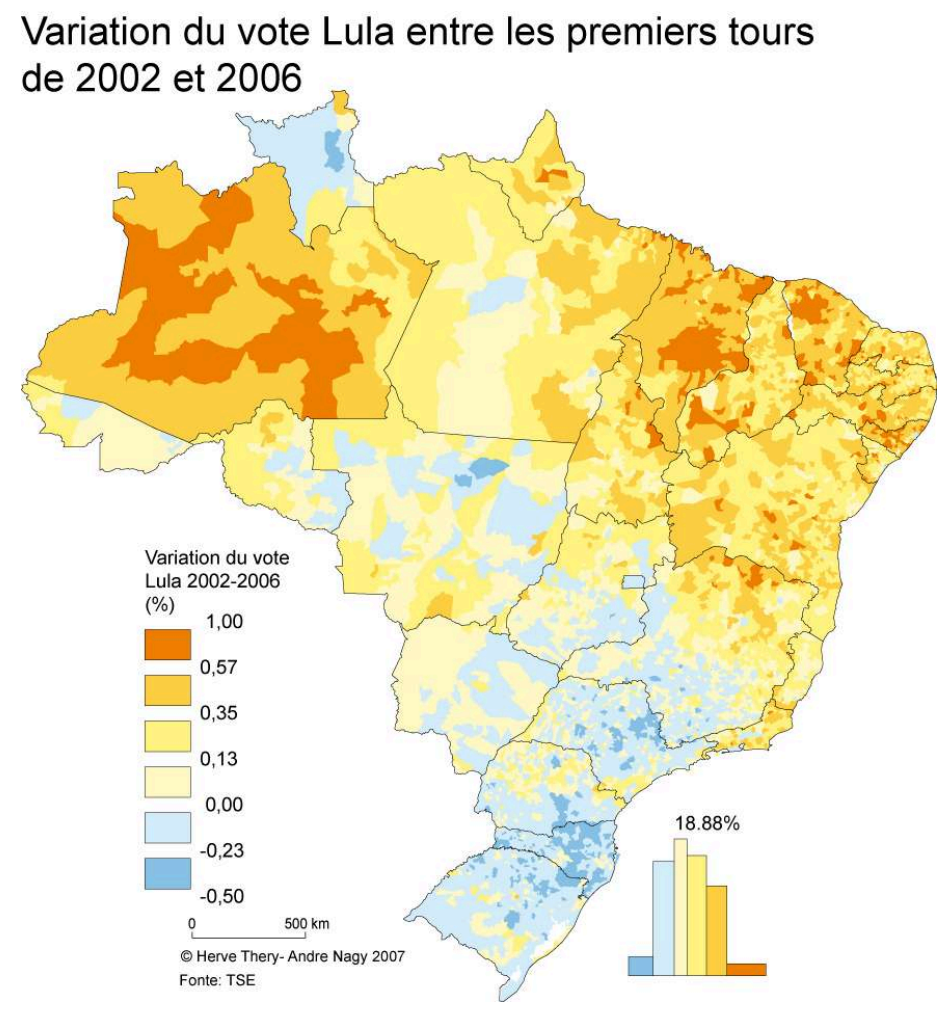
pas très cher - et avec l'argent du contribuable - mais on pourrait aussi dire - avec plus d'indulgence - que ce premier président issu des classes populaires, né dans le Nordeste, a rendu à «son peuple» ce qui lui était dû, après des siècles de spoliation. Pour préciser les choses - à charge ou à décharge - on peut indiquer les montants que représente la Bolsa familia : 8,3 milliards de Reais ( 3 milliards d'Euros), 0,36\% du PIB brésilien. L'opposition est évidemment furieuse, mais après tout, ceux qui ont monté ce «modèle de développement» si inégalitaire auraient bien dû penser qu'un jour ou l'autre, dans un système démocratique où tous les votes se valent, la masse des pauvres pourrait vouloir appuyer qui sait leur parler et lui donner un peu - si peu que soit - des fruits de la croissance économique, et qu'elle soutiendrait ensuite son bienfaiteur : cela s'appelle - littéralement dans certains cas - la reconnaissance du ventre.

Pour mieux cerner cet étonnant retournement de la base électorale entre 2002 et 2006, on a soumis les résultats des deux tours des deux élections à une analyse factorielle, en ne retenant que les deux principaux concurrents, Lula et Alckmin en 2006, Lula et Serra (aujourd'hui gouverneur de l'État de São Paulo) en 2002. 


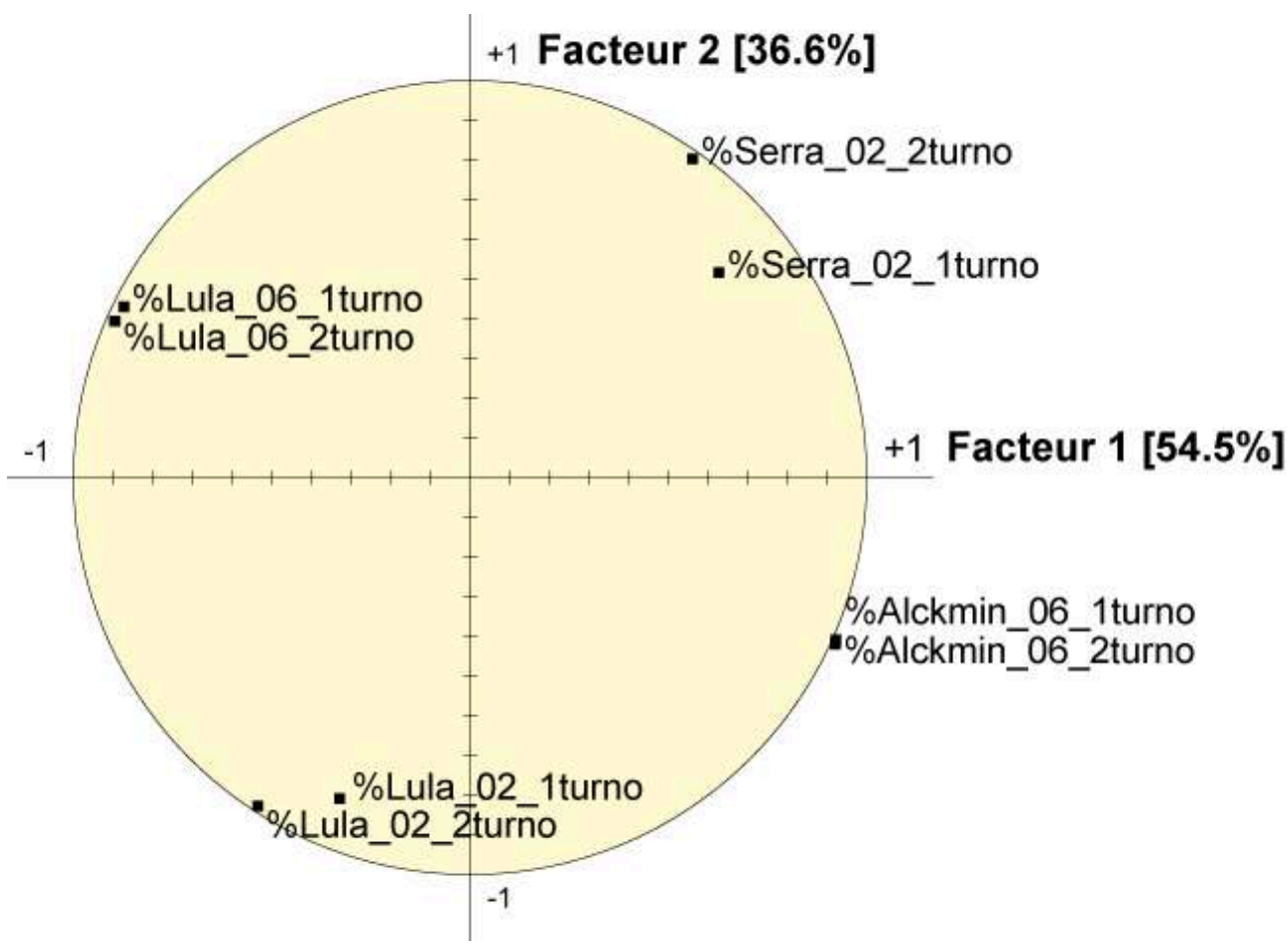

Rappelons brièvement le principe de ce type d'analyse : il vise à repérer les facteurs qui différencient la masse des données qui y est soumise, allant de celui qui représente la plus grande partie possible de leur variance (facteur 1$)$ aux suivants $(2,3$, etc.), qui apportent une part décroissante de celle-ci et donc de la différenciation des individus, en l'occurrence le vote pour tel ou tel candidat dans chaque commune brésilienne. Ces facteurs sont ensuite représentés sur un graphique cartésien les associant deux à deux, les proximités et oppositions des variables indiquant qu'elles jouent dans le même sens - ou en sens contraire - dans l'analyse des données.

Le plan factoriel associant les facteurs 1 et 2 (qui représentent ensemble $91 \%$ de la variance) présente une double opposition : l'axe 1 ( $54,5 \%$ de la variance) oppose bien Lula à ses adversaires de 2002 et 2006, mais l'axe 2 (36,6\% de la variance) l'oppose à luimême : si le Lula de 2002 est à l'opposé de Serra (sur l'axe vertical), le Lula de 2006 est (sur ce même axe) plus proche d'Alckmin que de lui-même en 2002, ou a fortiori de Serra.

Les cartes représentant les scores sur les axes 1 et 2 vont dans le même sens : celle de l'axe 1 sépare les régions qui ont élu Lula (couleurs froides) de celles qui ont préféré Alckmin (couleurs chaudes). Elle fait bien apparaître que le front pionnier du soja et de l'élevage, dans le Mato Grosso et le Pará, a voté massivement Alckmin, (en proportion du moins, car les effectifs en jeu sont faibles).

Celle de l'axe 2 oppose les régions qui étaient naguère favorables à Serra mais on voté cette fois Lula (couleurs chaudes, haute Amazonie et Nordeste) et celles qui ont basculé dans l'autre sens, de Lula à Alckmin, dans le Sud-Sudeste, le Centre Ouest et en Amazonie centrale. On remarque notamment le basculement des zones d'agriculture paysanne du Sud, aux confins du Santa Catarina et du Paraná, probablement déçues par l'option nette pour la grande agriculture d'exportation prise par Lula. 
Illustration 7

\section{Facteur 1}

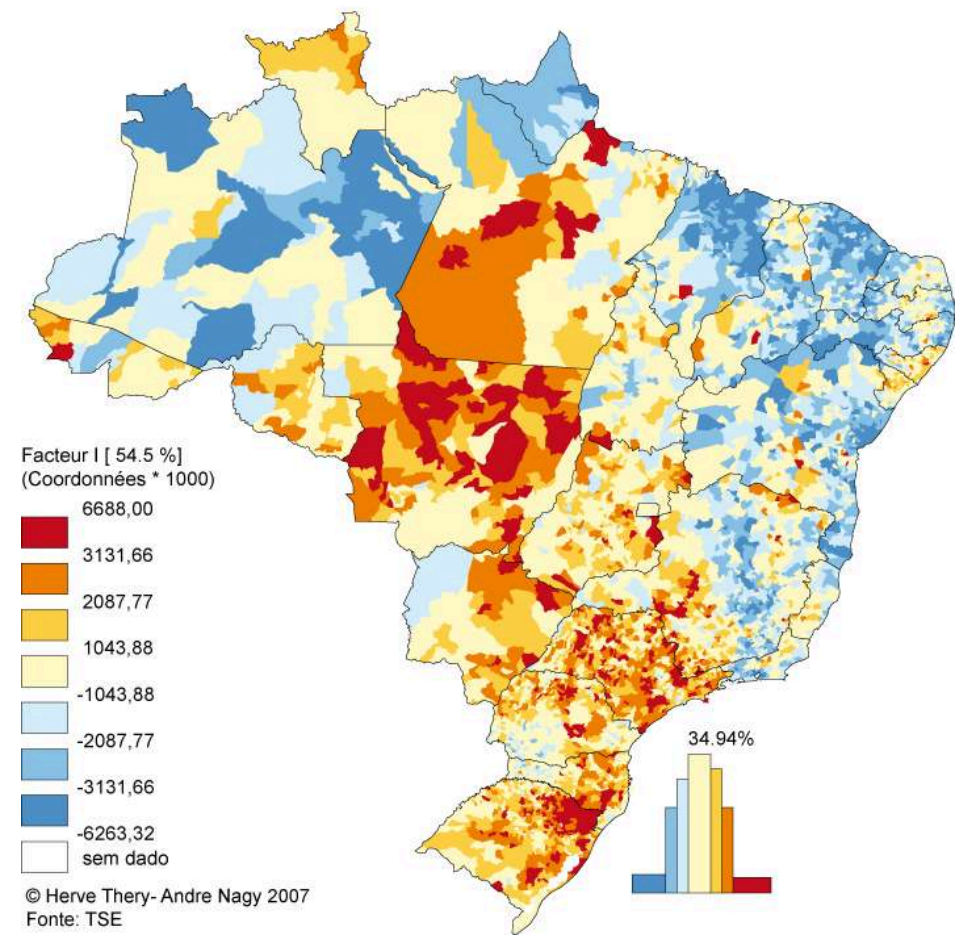

Illustration 8

\section{Facteur 2}

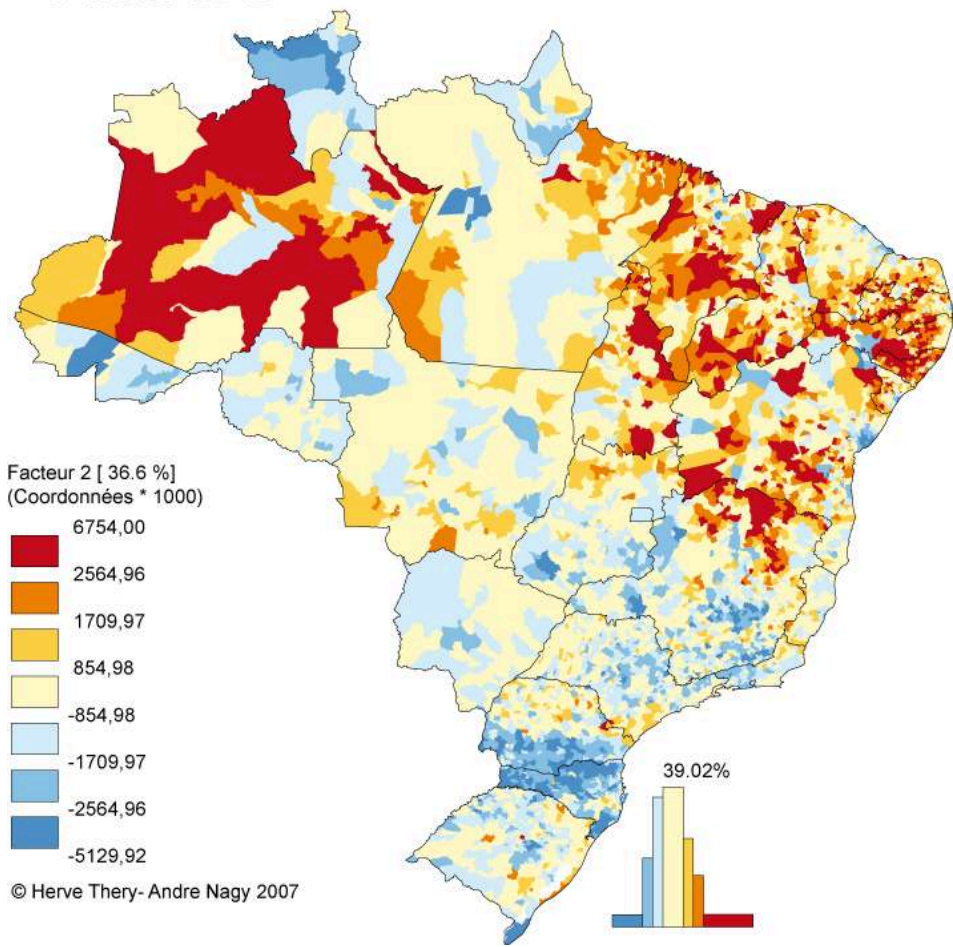

$17 \mathrm{Au}$ terme de cette analyse, pour confirmer davantage l'étroite coïncidence entre pauvreté et appui à Lula, on peut mettre en regard les communes où celui-ci est arrivé 
en tête et celles dont l'IDH (Indice de Développement Humain) est le plus bas. Élaboré par le PNUD pour comparer entre eux les pays plus ou moins développés, cet indice a été repris au Brésil pour à l'échelle de ses 5561 communes, en prenant donc en compte non seulement le revenu par tête, mais aussi l'éducation et la santé, ce qui est un des mérites essentiels de cet indice. Calculé pour 1970, 1980, 1991 et 2000, il souligne la situation précaire du Nordeste et de l'Amazonie, alors que le Sud et le Sudeste - à l'exception d'une région qui s'étend du sud-est de l'état de São Paulo au centre du Paraná - a de bien meilleurs résultats Le Centre-Ouest tend à lui ressembler de plus en plus, à mesure que les fronts pionniers avancent à travers les savanes centrales et - de plus en plus - la forêt amazonienne

Illustration 9 - Elections 2006 et indice de développement humain (DH)

Élection 2006 et indice de dévloppement humain (IDH)

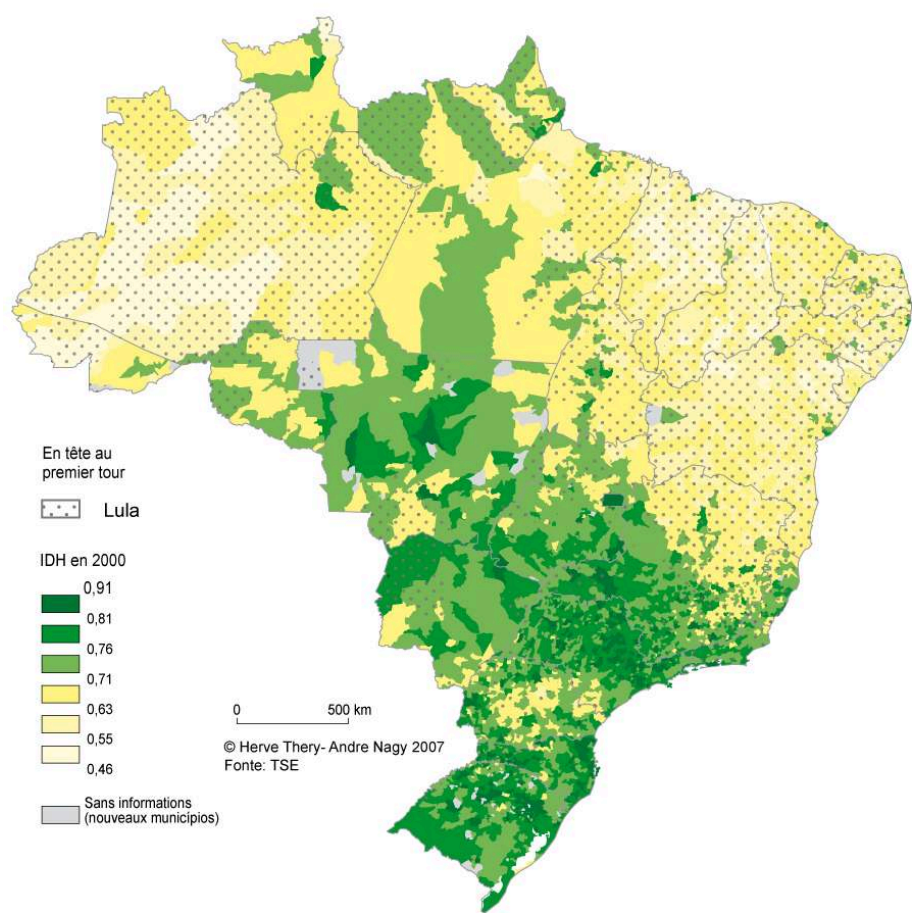

On pourrait, arrivé à ce point, se demander comment qualifier aujourd'hui ce gouvernement? De gauche? Les réformes auxquelles on reconnaît habituellement un gouvernement de gauche n'ont pas été très nombreuses, c'est le moins qu'on puisse dire : les projets de renforcement de la proportionnalité de l'impôt sur le revenu, de création de l'impôt sur la fortune ont été abandonnés dès la première année, et la politique économique suivie a été parfaitement orthodoxe : le FMI l'en a félicité et les agences qui produisent des classements destinés aux investisseurs internationaux, comme la Standard \& Poor's, ont amélioré la note du Brésil. Les mesures sociales favorisent bien les plus pauvres, mais on voit bien qu'elles aussi ont servi à asseoir la base politique du président. Le basculement à droite des régions pionnières est elle aussi assez logique, les entrepreneurs enrichis par la culture du soja n'ayant aucune raison de soutenir un président qui appuie la réforme agraire et tolère les invasions de terres du MST (Mouvement des sans terres). Populiste ? Sans doute, par exemple en ce que la relation directe au Président est plus forte que l'appui à son parti, éclaboussé par les scandales alors que Lula garde de hauts niveaux de popularité, et s'appuie de plus en 
plus à des partis dont moins qu'on puisse dire est que est que l'éthique n'est pas leur préoccupation dominante. Mais si populisme il y a, c'est dans le respect total du droit, sans aucun des effets de tribune et des coups de force d'un Chaves ou d'un Morales.

Une chose est sûre en tout cas, la situation politique brésilienne - et plus largement latino-américaine - entre mal dans nos catégories d'analyse européennes, et ceux qui voient le continent submergé par une "vague rose ", mettant Lula dans le même sac que Bachelet (Chili), Kirchner (Argentine), Chavez (Venezuela), Correa (Équateur) et Morales (Bolivie) sont bien imprudents, ou se consolent en projetant dans ce monde lointain et mythique leurs espoirs déçus par les frustrations de la vie politique nationale...

\section{BIBLIOGRAPHIE}

André Rodrigues Nagy, Hervé Théry, «Lula réélu, le retour aux origines ? », M@ppemonde n 84

(4-2006), http://mappemonde mgm fr/actualites/lula html

Paraná, Denise, Lula, o filho do Brasil, Editora Fundação Perseu Abramo, 2002

Tribunal Superior Eleitoral http://www tse gov br/internet/index html

\section{NOTES}

1. La première étape des cartes à base statistiques a été réalisée en utilisant le logiciel Philcarto (disponible sur le site http://perso.club-internet.fr/philgeo).

2. Il convient ici de rappeler qu'au Brésil le vote est obligatoire, sous peine d'amende et de graves difficultés administratives pour les électeurs défaillants. Cela explique l'absence de mention des abstentionnistes dans le tableau ci-dessus, l'impossibilité d'une abstention de protestation et l'importance du vote des régions sous-développées : dans un système de vote libre il est probable que les abstentions y seraient nombreuses, dans un système de vote obligatoire il est essentiel de les conquérir.

\section{RÉSUMÉS}

La réélection de Luiz Inácio da Silva, le 29 octobre 2006, malgré les scandales politico-financiers qui ont marqué les trois dernières années de son premier mandat est due en grande partie à un changement social et géographique de sa base électorale principale : appuyé jusque là par les régions industrielles du Sud, il a été cette fois porté par le Nord et le Nordeste, les régions pauvres du pays, comme le montre une série de cartes fondées sur l'analyse des résultats électoraux. 
The re-election of Luiz Inácio da Silva, on October 29, 2006, in spite of the politico-financial scandals which marked the three last years of its first mandate is due mainly to a social and geographical change of its principal electoral base: supported until there by the industrial areas of the South, it was this time carried by North and Nordeste, the poor areas of the country, as shown by a series of maps based on the analysis of the electoral results.

A reeleição de Luiz Inácio da Silva, no dia 29 de Outubro de 2006, apesar dos escândalos políticofinanceiros que marcaram os três últimos anos do seu primeiro mandato, é devido em grande parte à uma mudança social e geográfica da sua base eleitoral principal: apoiado até então pelas regiões industriais do Sul, foi esta vez levado pelo Norte e o Nordeste, as regiões pobres do país, como mostra-o uma série de mapas fundados na análise dos resultados eleitorais.

\section{INDEX}

Mots-clés : Brésil, cartographie, élection, Lula

Keywords : Brazil, cartography, elections, Lula

\section{AUTEURS}

\section{HERVÉ THÉRY}

Hervé Théry (hthery@aol.com) est directeur de recherche au CNRS et professor convidado na USP, Catedra Pierre Monbeig. Il a notamment publié : Le Brésil, Armand Colin, 5e édition ; Atlas do Brasil, avec Neli Aparecida de Mello, Edusp.

\section{ANDRÉ NAGY}

André Nagy (arnagy@seade.gov.br), sociologue, Analista de projetos na Fundação Seade, Pos graduando em geografia humana na USP, Atlas Seade da Economia Paulista, $2006 \mathrm{em}$ www.seade.gov.br, Municipio em Mapas, Prefeitura de São Paulo, 2006. 